\title{
Recurrent acute portal vein thrombosis in liver cirrhosis treated by rivaroxaban
}

\author{
Hyeyoung Yang, Seo Ree Kim, and Myeong Jun Song \\ Division of Hepatology, Department of Internal Medicine, Daejeon St. Mary's Hospital, College of Medicine, The Catholic University of \\ Korea, Daejeon, Korea
}

Cirrhosis can occur with the development of portal vein thrombosis (PVT). PVT may aggravate portal hypertension, and it can lead to hepatic decompensation. The international guideline recommends for anticoagulation treatment to be maintained for at least 3 months in all patients with acute PVT. Low-molecular-weight-heparin and changing to warfarin is the usual anticoagulation treatment. However, warfarin therapy is problematic due to a narrow therapeutic window and the requirement for frequent dose adjustment, which has prompted the development of novel oral anticoagulants for overcoming these problems. We report a 63-year-old female who experienced complete resolution of recurrent acute PVT in liver cirrhosis after treatment with rivaroxaban. (Clin Mol Hepatol 2016;22:499-502)

Keywords: Liver cirrhosis; Portal vein thrombosis; Rivaroxaban

\section{INTRODUCTION}

Liver cirrhosis is a risk factor for the development of portal vein thrombosis (PVT). Anticoagulant therapy for at least 3 months is generally recommended for recanalization to prevent intestinal infarction and worsening of portal hypertension, ascites and jaundice. Low-molecular-weight-heparin and warfarin contribute the traditional anticoagulation protocol. However, warfarin has a narrow therapeutic window and carries the risk of drug interactions, and requires frequent dose adjustments. Therefore, novel oral anticoagulants (NOACs) have been developed to compensate for the troublesome aspects of warfarin therapy.

Rivaroxaban is a promising therapeutic option to treat PVT in cirrhosis. Rivaroxaban is an oral anticoagulant agent of factor $\mathrm{Xa}$ inhibitor class. Oral rivaroxaban can be prescribed in a fixed dosage without coagulation monitoring. We report a case of com- plete recanalization for recurrent acute PVT in a cirrhotic patient who was treated with rivaroxaban.

\section{CASE REPORT}

A 63-year-old female with liver cirrhosis was incidentally diagnosed with PVT on liver dynamic computed tomography (CT). She was asymptomatic and there were no signs to indicate infection, including fever or leukocytosis. The laboratory findings were as follows: WBC count, 3,100/ $\mathrm{mm}^{3}$; hemoglobin, $12.6 \mathrm{~g} / \mathrm{dL}$; platelet

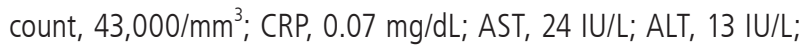
ALP, 77 IU/L; GGT, 12 IU/L; total bilirubin, $0.7 \mathrm{mg} / \mathrm{dL}$; albumin, 3.5 $\mathrm{g} / \mathrm{dL} ;$ PT INR, 1.13, and alpha-fetoprotein (AFP) $1.12 \mathrm{ng} / \mathrm{mL}$. She was Child-Pugh class of $A$ and had a score of 9 on the patient's Model for End-stage Liver disease (MELD). Viral study showed

\footnotetext{
Abbreviations:

AASLD, American Association for the Study of Liver Disease; AFP, alpha-fetoprotein; ALP, alkaline phosphatase; ALT, alanine aminotransferase; anti HCV Ab, anti-hepatitis $C$ virus antibody; $A S T$, aspartate aminotransferase; CRP, C-reactive protein; $C T$, computed tomography; CTP, Child-Turcotte-Pugh; FDP, fibrinogen degradation product; GGT, gamma-glutamyl transpeptidase; HBsAg, hepatitis B surface antigen; HBV DNA, hepatitis B virus deoxyribonucleic acid; INR, international normalized ratio; MELD, Model for End-stage Liver disease; NOACs, novel oral anticoagulants; PVT, portal vein thrombosis; WBC, White blood cell
}

\section{Corresponding author : Myeong Jun Song}

Division of Hepatology, Department of Internal Medicine, Daejeon St. Mary's Hospital, College of Medicine, The Catholic University of Korea, 64, Daeheung-ro, Jung-gu, Daejeon 34943, Korea

Tel. +82-42-220-9291, Fax. +82-42-252-6807

E-mail; mjsong95@gmail.com 


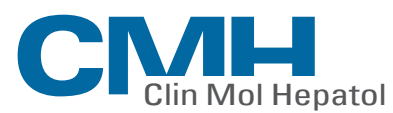

Volume 22 Number_4 December 2016
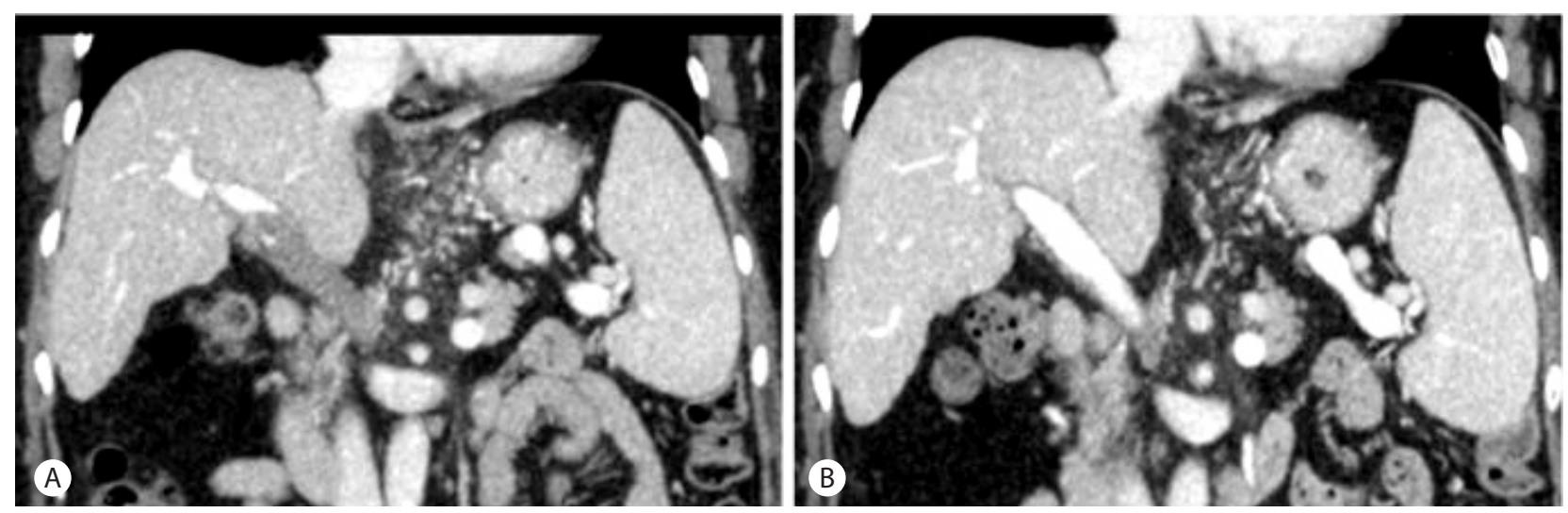

Figure 1. Contrast-enhanced liver dynamic CT in the portal venous phase yielded coronal images showing acute PVT. (A) CT image showing hypoattenuated filling defects in the main portal vein. (B) CT image showing complete resolution of acute PVT after 3 months of treatment with warfarin. CT, computed tomography; PVT, portal vein thrombosis.

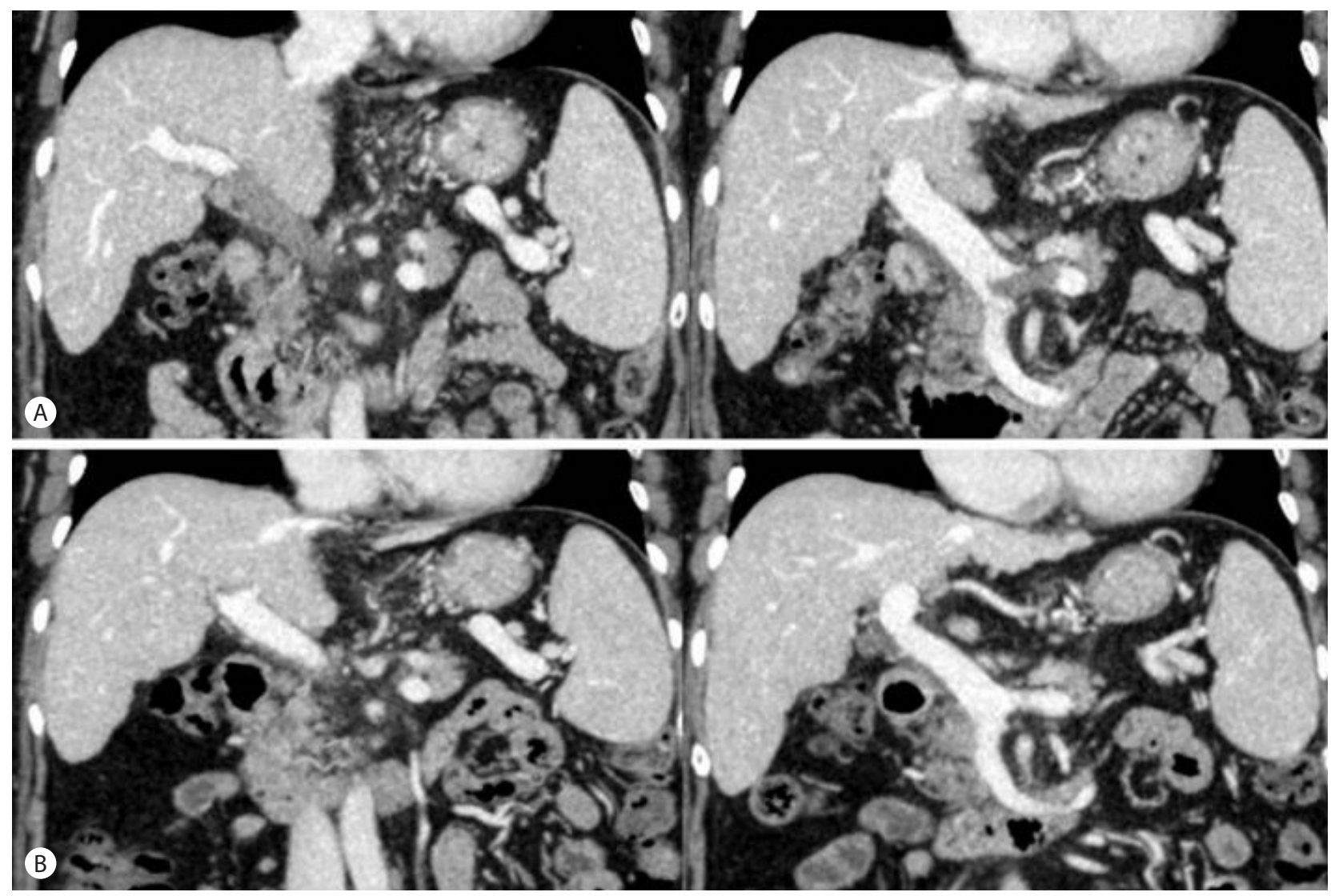

Figure 2. Contrast-enhanced liver dynamic CT in the portal venous phase yielded coronal images showing recurrent PVT. (A) CT image showing recurrent PVT in the main portal vein. (B) CT image showing resolution of recurrent PVT after 3 months of treatment with rivaroxaban. CT, computed tomography; PVT, portal vein thrombosis.

HBsAg positive, HBsAb negative, HBeAg negative, HBV DNA at $1.16 \times 10^{2}$ copies $/ \mathrm{mL}$ and anti-HCV Ab negative. Coagulation tests confirmed a low level of protein C activity, 66\% (70-130\%). Other coagulation laboratory findings were as follows: protein $\mathrm{S}$ activity, 56\% (55-123\%); protein S (free), 93.5\% (50-150\%); protein S (total), 62.8\% (60-150\%); antithrombin III, 59.1\% (80120\%); fibrinogen (functional), $194 \mathrm{mg} / \mathrm{dL}(200-400) ; \mathrm{FDP}, 8.6$ $\mu \mathrm{g} / \mathrm{mL}(0-5)$, and factor $\mathrm{V}$ Leiden, negative. 
Hyeyoung Yang, et al.

Novel oral anticoagulants

On baseline liver dynamic CT, hypo-attenuated filling defects were visible in the right portal vein and main portal vein, suggesting thrombosis (Fig. 1A). Malignant PVT was excluded due to the lack of enhancement of the thrombus on dynamic CT during the arterial phase and a normal AFP level. For recanalization of the portal vein, we began with low molecular weight heparin (Enoxaparin $1 \mathrm{mg} / \mathrm{kg}$ via subcutaneous injection) and changed to warfarin. We maintained anticoagulation therapy for 3 months. On follow-up liver dynamic CT, resolution of the PVT was identified (Fig. 1B). After resolution of the PVT, we discontinued warfarin treatment. Follow-up liver dynamic CT 3 months after cessation of warfarin showed a recurrence of PVT (Fig. 2A). At that time, the laboratory findings were as follows: total bilirubin, $1.1 \mathrm{mg} / \mathrm{dL}$ : albumin, $4.1 \mathrm{~g} / \mathrm{dL}$, and PT INR, 1.13. Child-Pugh class and MELD score were $A$ and 8 , respectively. We then initiated rivaroxaban instead of warfarin (15 mg twice per day for 3 weeks and then, $20 \mathrm{mg}$ per day). The dosage of rivaroxaban can be altered as needed to treat acute deep vein thrombosis (DVT) and PTE. ${ }^{1}$ Three months after beginning rivaroxaban, follow-up liver dynamic CT showed resolution of the PVT (Fig. 2B). Six months after beginning rivaroxaban, follow-up liver dynamic CT still showed full resolution of the PVT. During the rivaroxaban treatment, the patient did not experience any adverse events, including gastrointestinal bleeding.

\section{DISCUSSION}

Cirrhotic patients are at risk for PVT, which has accounted for $5-30 \%$ of patients with portal hypertension. ${ }^{2,3}$ The Incidence of PVT in advanced liver cirrhosis is high, at less than $1 \%$ in patients with compensated cirrhosis, but 8-25\% in liver transplantation candidates due to venous stasis and their hypercoagulable status. ${ }^{4,5}$

PVT in cirrhotic patients is often accompanied by gastrointestinal bleeding including variceal bleeding, intestinal infarction, portal hypertension, and portal cholangiopathy. ${ }^{6}$ When the thrombus extends to the superior mesenteric vein, the risk of intestinal infarction is high. ${ }^{6}$ Cirrhotic patients with chronic PVT can develop portal hypertension, which leads to the formation of collaterals around the obstructed portal vein, aggravation of ascites, and varix. Portal cholangiopathy may be seen in patients with longstanding PVT and can cause signs of biliary obstruction, such as pruritus, obstructive jaundice. ${ }^{7,8}$ PVT Complications can be quite serious, requiring prompt medical attention and treatment.

Rivaroxaban is a new treatment option for PVT in patients with cirrhosis. It is an oral anticoagulative agent that inhibits free Factor $\mathrm{Xa}$ and Factor $\mathrm{Xa}$ bound in the prothrombinase complex. ${ }^{9}$ Factor Xa catalyzes the conversion of prothrombin to thrombin, and one molecule of Factor $X a$ generates over 1,000 thrombin molecules. ${ }^{10}$ Thus, inhibiting Factor Xa can block this burst of thrombin generation, thereby diminishing thrombin-mediated activation of coagulation and platelets. ${ }^{11}$

Rivaroxaban therapy has some advantages compared with warfarin. First, rivaroxaban can be prescribed without dose modification and without coagulation monitoring. Absorption of rivaroxaban is also unaffected by food. ${ }^{12}$ Warfarin requires highly variable dose adjustments, necessitating close monitoring. Second, rivaroxaban also has a faster onset time than warfarin therapy; the onset time for rivaroxaban is $30 \mathrm{~min}$, compared with an onset time of 36-72 hours for warfarin, which must be combined with heparin. ${ }^{13}$ The discontinuation period of NOACs is relatively short; furthermore, the half life of warfarin is 20-60 hours, compared with 5-9 hours in young adults and 11-13 hours in older adults for rivaroxaban. ${ }^{14}$ Therefore, prior to an invasive procedure or elective surgery, warfarin should be stopped for 5 days previously, but rivaroxaban must be stopped only 1-2 days prior if there is no bleeding evidence. Compared to traditional anticoagulation therapy, NOACs have similar safety characteristics in cirrhotic patients. ${ }^{15}$

However, rivaroxaban is contraindicated in the case of patients with impaired hepatic function (Child-Pugh classification B, C). Although rivaroxaban has limited use for the treatment of PVT in cirrhotic patients, there are some case reports of PVT controlled by rivaroxaban treatment. ${ }^{16-18}$

Another important issue of this case is the optimal duration of anticoagulation therapy for acute PVT. Anticoagulation therapy has proven benefits in patients with acute DVT. ${ }^{14}$ The American Association for the Study of Liver Disease (AASLD) guideline recommends that anticoagulation treatment be maintained for at least 3 months for all patients with acute PVT. The relapse rate of venous thromboembolism is $12-18 \%$ at 2 years after treatment. ${ }^{19,20}$ In our case, we maintained traditional anticoagulation therapy for 3 months and achieved complete recanalization of portal vein. However, after 3 month from cessation of warfarin, PVT recurred. We then began rivaroxaban and have maintained NOACs since resolution of the PVT. However, there is no consensus on maintaining anticoagulation to prevent re-thrombosis. ${ }^{21}$

In conclusion, we reported a case of complete resolution of recurrent PVT in a cirrhotic patient who was treated with rivaroxaban. This case highlights that rivaroxaban could be an alternative option to traditional warfarin therapy. 


\section{Conflicts of Interest}

The authors have no conflicts to disclose.

\section{REFERENCES}

1. EINSTEIN Investigators, Bauersachs R, Berkowitz SD, Brenner B, Buller HR, Decousus $H$, et al. Oral rivaroxaban for symptomatic venous thromboembolism. N Engl J Med 2010;363:2499-2510.

2. Valla DC, Condat B, Lebrec D. Spectrum of portal vein thrombosis in the West. J Gastroenterol Hepatol 2002;17(Suppl 3):S224-S227.

3. Sarin SK, Sollano JD, Chawla YK, Amarapurkar D, Hamid S, Hashizume $\mathrm{M}$, et al. Consensus on extra-hepatic portal vein obstruction. Liver Int 2006;26:512-519.

4. Okuda K, Ohnishi K, Kimura K, Matsutani S, Sumida M, Goto N, et al. Incidence of portal vein thrombosis in liver cirrhosis. An angiographic study in 708 patients. Gastroenterology 1985;89:279-286.

5. Francoz C, Belghiti J, Vilgrain V, Sommacale D, Paradis V, Condat B, et al. Splanchnic vein thrombosis in candidates for liver transplantation: usefulness of screening and anticoagulation. Gut 2005;54:691-697.

6. Amitrano L, Guardascione MA, Brancaccio V, Margaglione M, Manguso $F$, lannaccone $L$, et al. Risk factors and clinical presentation of portal vein thrombosis in patients with liver cirrhosis. J Hepatol 2004;40:736-741.

7. Condat B, Vilgrain V, Asselah T, O'Toole D, Rufat P, Zappa M, et al. Portal cavernoma-associated cholangiopathy: a clinical and MR cholangiography coupled with MR portography imaging study. Hepatology 2003;37:1302-1308.

8. Sarin S, Bhatia V, Makwane U. Portal biliopathy in extrahepatic portal vein obstruction. Indian J Gastroenterol 1992;2:A82.

9. Roehrig S, Straub A, Pohlmann J, Lampe T, Pernerstorfer J, Schlemmer $\mathrm{KH}$, et al. Discovery of the Novel Antithrombotic Agent 5-Chloro-N-(\{(5S)-2-oxo-3- [4-(3-oxomorpholin-4-yl)phenyl]-1,3oxazolidin-5-yl\}methyl)thiophene- 2-carboxamide (BAY 59-7939): An Oral, Direct Factor Xa Inhibitor. J Med Chem 2005;48:59005908.

10. Mann KG, Brummel K, Butenas S. What is all that thrombin for? J
Thromb Haemost 2003;1:1504-1514.

11. Perzborn E, Strassburger J, Wilmen A, Pohlmann J, Roehrig S, Schlemmer $\mathrm{KH}$, et al. In vitro and in vivo studies of the novel antithrombotic agent BAY 59-7939--an oral, direct Factor Xa inhibitor. J Thromb Haemost 2005;3:514-521.

12. Smythe MA, Fanikos J, Gulseth MP, Wittkowsky AK, Spinler SA, Dager WE, et al. Rivaroxaban: practical considerations for ensuring safety and efficacy. Pharmacotherapy 2013;33:1223-1245.

13. Holbrook AM, Pereira JA, Labiris R, McDonald H, Douketis JD, Crowther $M$, et al. Systematic overview of warfarin and its drug and food interactions. Arch Intern Med 2005;165:1095-1106.

14. Bates SM, Ginsberg JS. Clinical practice. Treatment of deep-vein thrombosis. N Engl J Med 2004;351:268-277.

15. Intagliata NM, Henry ZH, Maitland $H$, Shah NL, Argo CK, Northup $P G$, et al. Direct Oral Anticoagulants in Cirrhosis Patients Pose Similar Risks of Bleeding When Compared to Traditional Anticoagulation. Dig Dis Sci 2016;61:1721-1727.

16. Lenz K, Dieplinger B, Buder R, Piringer P, Rauch M, Voglmayr M. Successful treatment of partial portal vein thrombosis (PVT) with low dose rivaroxaban. Z Gastroenterol 2014;52:1175-1177.

17. Pannach S, Babatz J, Beyer-Westendorf J. Successful treatment of acute portal vein thrombosis with rivaroxaban. Thromb Haemost 2013;110:626-627.

18. Martinez M, Tandra A, Vuppalanchi R. Treatment of acute portal vein thrombosis by nontraditional anticoagulation. Hepatology 2014;60:425-426.

19. Pabinger I, Brucker S, Kyrle PA, Schneider B, Korninger HC, Niessner $H$, et al. Hereditary deficiency of antithrombin III, protein $C$ and protein $\mathrm{S}$ : prevalence in patients with a history of venous thrombosis and criteria for rational patient screening. Blood Coagul Fibrinolysis 1992;3:547-553.

20. Hansson PO, Sorbo J, Eriksson H. Recurrent venous thromboembolism after deep vein thrombosis: incidence and risk factors. Arch Intern Med 2000;160:769-774.

21. Primignani $M$, Tosetti $G$, La Mura V. Therapeutic and clinical aspects of portal vein thrombosis in patients with cirrhosis. World J Hepato 2015;7:2906-2912. 